\title{
On $\vec{p}(x)$-Anisotropic Problems with Neumann Boundary Conditions
}

\begin{abstract}
Anass Ourraoui
Department of Mathematics (ENSAH), Nonlinear Analysis Laboratory (FSO), University of Mohammed First, 60000 Oujda, Morocco

Correspondence should be addressed to Anass Ourraoui; anas.our@hotmail.com

Received 6 July 2015; Revised 8 November 2015; Accepted 23 November 2015

Academic Editor: Emmanuel Hebey

Copyright (c) 2015 Anass Ourraoui. This is an open access article distributed under the Creative Commons Attribution License, which permits unrestricted use, distribution, and reproduction in any medium, provided the original work is properly cited.

This work is devoted to the study of a general class of anisotropic problems involving $\vec{p}(\cdot)$-Laplace operator. Based on the variational method, we establish the existence of a nontrivial solution without Ambrosetti-Rabinowitz type conditions.
\end{abstract}

\section{Introduction}

The elliptic problems in anisotropic form concerning the Sobolev space with variable exponents have recently attracted the attention of many mathematicians; see [1-13] and the references therein. Such equations arise in connection with the equations describing electromagnetic fields and the plasma physics; see $[14,15]$ and various applications like those in thermorheological fluids [16], elastic mechanics [17], and image restoration [18]. They also appear in biology; see, for instance, Bendahmane et al. in [19], as a model for the propagation of epidemic diseases in heterogeneous domains.

In the present paper, we study the anisotropic nonlinear elliptic problem of the form

$$
\begin{aligned}
-\sum_{i=1}^{N} \partial_{x_{i}} a_{i}\left(x, \partial_{x_{i}} u\right)+|u|^{p_{M}(x)-2} u & =f(x, u), \\
& \text { for } x \in \Omega, \\
\sum_{i=1}^{N} a_{i}\left(x, \partial_{x_{i}} u\right) v_{i} & =0 \quad \text { for } x \in \partial \Omega,
\end{aligned}
$$

where $\Omega \subset \mathbb{R}^{N}(N \geq 2)$ is a bounded open set with smooth boundary and $v_{i}$ are the components of the outer normal unit vector and for $i \in\{1, \ldots, N\}$, for all $x \in \bar{\Omega}, p_{M}=$ $\max \left\{p_{1}(x), \ldots, p_{N}(x)\right\}$, where the exponents $p_{i}: \bar{\Omega} \rightarrow \mathbb{R}$ are continuous functions such that $\inf _{x \in \Omega} p_{i}(x)>1$.
We assume that the functions $f$ and $a_{i}: \Omega \times \mathbb{R}^{N} \rightarrow \mathbb{R}$ are Carathéodory and satisfying the following conditions for all $i \in\{1,2, \ldots, N\}$.

(A1) There exists a positive constant $\bar{c}_{i}$ such that $a_{i}$ fulfills

$$
\left|a_{i}(x, \xi)\right| \leq \bar{c}_{i}\left(b_{i}(x)+|\xi|^{p_{i}(x)-1}\right),
$$

for all $x \in \Omega$ and all $\xi \in \mathbb{R}^{N}$, where $b_{i} \in L^{p_{i}^{\prime}(\cdot)}(\Omega)$ (with $\left.1 / p_{i}(x)+1 / p_{i}^{\prime}(x)=1\right)$ is a nonnegative function and $A_{i}: \Omega \times \mathbb{R}^{N} \rightarrow \mathbb{R}$ is the mapping which verifies

$$
A_{i}(x, \xi)=\int_{0}^{\xi_{i}} a_{i}\left(x, \xi_{1}, \ldots, \xi_{i-1}, s, \xi_{i+1}, \ldots, \xi_{N}\right) d s .
$$

(A2) There exists $d_{i}>0$ such that

$$
d_{i}|\xi|^{p_{i}(x)} \leq p_{i}(x) A_{i}(x, \xi)
$$

for all $x \in \Omega$ and all $\xi \in \mathbb{R}$.

(A3) The monotonicity condition

$$
\left[a_{i}(x, s)-a_{i}(x, t)\right](s-t)>0
$$

takes place for all $x \in \Omega$ and all $s, t \in \mathbb{R}$ with $s \neq t$.

Example 1. We take

$$
a_{i}(x, s)=|s|^{p_{i}(x)-2} s, \quad \forall i \in\{1, \ldots, N\},
$$


and then the operator

$$
\sum_{i=1}^{N} \partial_{x_{i}} a_{i}\left(x, \partial_{x_{i}} u\right)
$$

becomes in particular $\vec{p}(\cdot)$-Laplace operator

$$
\Delta_{\vec{p}(x)}(u)=\sum_{i=1}^{N} \partial_{x_{i}}\left(\left|\partial_{x_{i}} u\right|^{p_{i}(x)-2} \partial_{x_{i}} u\right) .
$$

This is why operators (7) are often known as generalized $\vec{p}(\cdot)$ Laplace type operators.

On the other hand, the anisotropic equations with the variable exponent growth conditions enable the study of equations with more complicated nonlinearities since the differential operator $\Delta_{\vec{p}(x)}(u)$ allows a distinct behavior for partial derivatives in various directions.

This paper is organized as follows. In Section 2, we give the necessary notations; we also include some useful results involving the variable exponent Sobolev spaces in order to facilitate the reading of the paper. Finally, in Section 3, we prove the existence of nontrivial solution.

\section{Preliminaries and Main Result}

We introduce the setting of our problem with some auxiliary results. For convenience, we only recall some basic facts which will be used later; we refer to [20, 21].

For $r \in C_{+}(\bar{\Omega})$, we introduce the Lebesgue space with variable exponent defined by

$$
L^{r(\cdot)}(\Omega)=\{u:
$$

$u$ is a measurable real-valued function,

$$
\left.\int_{\Omega}|u(x)|^{r(x)} d x<\infty\right\}
$$

where

$$
C_{+}(\bar{\Omega})=\left\{r \in C(\bar{\Omega} ; \mathbb{R}): \inf _{x \in \Omega} r(x)>1\right\} .
$$

This space, endowed with the Luxemburg norm,

$$
\|u\|_{L^{r(\cdot)}(\Omega)}=\inf \left\{\mu>0: \int_{\Omega}\left|\frac{u(x)}{\mu}\right|^{r(x)} d x \leq 1\right\}
$$

is a separable and reflexive Banach space. We also have an embedding result.

Proposition 2. Assume that $\Omega$ is bounded and $r_{1}, r_{2} \in C_{+}(\bar{\Omega})$ such that $r_{1} \leq r_{2}$ in $\Omega$. Then, the embedding $L^{r_{2}(\cdot)}(\Omega) \hookrightarrow$ $L^{r_{1}(\cdot)}(\Omega)$ is continuous.

Furthermore, the Hölder-type inequality

$$
\left|\int_{\Omega} u(x) v(x) d x\right| \leq 2\|u\|_{L^{r \cdot(\cdot)}(\Omega)}\|v\|_{L^{r^{\prime}(\cdot)}(\Omega)}
$$

holds for all $u \in L^{r(\cdot)}(\Omega)$ and $v \in L^{r^{\prime}(\cdot)}(\Omega)$, where $L^{r^{\prime}(\cdot)}(\Omega)$ is the conjugate space of $L^{r(\cdot)}(\Omega)$, with $1 / r(x)+1 / r^{\prime}(x)=1$.

Moreover, we denote

$$
\begin{aligned}
& r^{+}=\sup _{x \in \Omega} r(x), \\
& r^{-}=\inf _{x \in \Omega} r(x)
\end{aligned}
$$

and, for $u \in L^{r(\cdot)}(\Omega)$, we have the following properties:

$$
\begin{aligned}
\|u\|_{L^{r \cdot()}(\Omega)} & <1 \quad(=1 ;>1) \Longleftrightarrow \\
\int_{\Omega}|u(x)|^{r(x)} d x & <1 \quad(=1 ;>1) ; \\
\|u\|_{L^{r \cdot()}(\Omega)}>1 \Longrightarrow & \\
\|u\|_{L^{r(\cdot)}(\Omega)}^{r^{-}} & \leq \int_{\Omega}|u(x)|^{r(x)} d x \leq\|u\|_{L^{r(\cdot)}(\Omega)}^{r^{+}} ; \\
\|u\|_{L^{r(\cdot)}(\Omega)} & <1 \Longrightarrow \\
\|u\|_{L^{r(\cdot)}(\Omega)}^{r^{+}} & \leq \int_{\Omega}|u(x)|^{r(x)} d x \leq\|u\|_{L^{r(\cdot)}(\Omega)}^{r^{-}} ; \\
\|u\|_{L^{r(\cdot)}(\Omega)} & \longrightarrow 0(\longrightarrow \infty) \Longleftrightarrow \\
\int_{\Omega}|u(x)|^{r(x)} d x & \longrightarrow 0(\longrightarrow \infty) .
\end{aligned}
$$

To recall the definition of the isotropic Sobolev space with variable exponent, $W^{1, r(\cdot)}(\Omega)$, we set

$$
\begin{aligned}
& W^{1, r(\cdot)}(\Omega) \\
& \quad=\left\{u \in L^{r(\cdot)}(\Omega): \partial_{x_{i}} u \in L^{r(\cdot)}(\Omega), \forall i \in\{1, \ldots, N\}\right\},
\end{aligned}
$$

endowed with the norm

$$
\|u\|_{W^{1, r(\cdot)}(\Omega)}=\|u\|_{L^{r(\cdot)}(\Omega)}+\sum_{i=1}^{N}\left\|\partial_{x_{i}} u\right\|_{L^{r(\cdot)}(\Omega)} .
$$

The space $\left(W^{1, r(\cdot)}(\Omega),\|\cdot\|_{W^{1, r(\cdot)}(\Omega)}\right)$ is a separable and reflexive Banach space.

Now, we consider $\vec{p}: \bar{\Omega} \rightarrow \mathbb{R}^{N}$ to be the vectorial function

$$
\vec{p}(x)=\left(p_{1}(x), \ldots, p_{N}(x)\right)
$$

with $p_{i} \in C_{+}(\bar{\Omega})$ for all $i \in\{1, \ldots, N\}$ and we put

$$
\begin{aligned}
& p_{M}(x)=\max \left\{p_{1}(x), \ldots, p_{N}(x)\right\}, \\
& p_{m}(x)=\min \left\{p_{1}(x), \ldots, p_{N}(x)\right\} .
\end{aligned}
$$

The anisotropic space with variable exponent is

$$
\begin{aligned}
X & =W^{1, \vec{p}(\cdot)}(\Omega)=\left\{u \in L^{p_{M}(\cdot)}(\Omega): \partial_{x_{i}} u\right. \\
& \left.\in L^{p_{i}(\cdot)}(\Omega), \quad \forall i \in\{1, \ldots, N\}\right\}
\end{aligned}
$$


and it is endowed with the norm

$$
\|u\|=\|u\|_{W^{1, \vec{p}^{(\cdot)}(\Omega)}}=\|u\|_{L^{p^{(\cdot)}(\Omega)}}+\sum_{i=1}^{N}\left\|\partial_{x_{i}} u\right\|_{L^{p^{(\cdot)}(\Omega)}} .
$$

The space $\left(W^{1, \vec{p}(\cdot)}(\Omega),\|\cdot\|_{W^{1, \vec{p}^{(\cdot)}}(\Omega)}\right)$ is a reflexive Banach space. Furthermore, an embedding theorem takes place for all the exponents that are strictly less than a variable critical exponent, which is introduced with the help of the notations

$$
\begin{aligned}
\bar{p}(x) & =\frac{N}{\sum_{i=1}^{N} 1 / p_{i}(x)}, \\
r^{\star}(x) & = \begin{cases}\frac{N r(x)}{[N-r(x)]}, & \text { if } r(x)<N, \\
\infty, & \text { if } r(x) \geq N .\end{cases}
\end{aligned}
$$

Proposition 3. Let $\Omega \subset \mathbb{R}^{N}$ be a bounded open set for all $i=1,2, \ldots, N$ and $p_{i} \in C_{+}(\bar{\Omega})$ for all $i \in\{1, \ldots, N\}$. If $q \in C(\bar{\Omega} ; \mathbb{R}), 1 \leq q(x)<\max \left\{\bar{p}^{*}(x), p_{M}(x)\right\}$ for all $x \in \bar{\Omega}$, then one has the compact and continuous embedding $W^{1, \vec{p}^{(\cdot)}}(\Omega) \hookrightarrow L^{q(\cdot)}(\Omega)$.

Remark 4. We make the following notations:

$$
\begin{aligned}
& \mathscr{F}_{1}=\left\{i \in\{1, \ldots, N\}:\left\|\partial_{x_{i}} u_{n}\right\|_{L^{p^{(} \cdot()}(\Omega)} \leq 1\right\}, \\
& \mathscr{F}_{2}=\left\{i \in\{1, \ldots, N\}:\left\|\partial_{x_{i}} u_{n}\right\|_{L^{p^{(}(\cdot)}(\Omega)}>1\right\} .
\end{aligned}
$$

Then, by (14), (15), and (16),

$$
\begin{aligned}
\sum_{i=1}^{N} \int_{\Omega}\left|\partial_{x_{i}} u_{n}\right|^{p_{i}(x)} d x= & \sum_{i \in \mathscr{F}_{1}} \int_{\Omega}\left|\partial_{x_{i}} u_{n}\right|^{p_{i}(x)} d x \\
& +\sum_{i \in \mathscr{F}_{2}} \int_{\Omega}\left|\partial_{x_{i}} u_{n}\right|^{p_{i}(x)} d x \\
\geq & \sum_{i \in \mathscr{F}_{1}}\left\|\partial_{x_{i}} u_{n}\right\|_{L^{p_{i}(\cdot)}}^{p^{+}} \\
& +\sum_{i \in \mathscr{F}_{2}}\left\|\partial_{x_{i}} u_{n}\right\|_{L^{p_{i}(\cdot)}}^{p_{m}^{-}} \\
\geq & \sum_{i=1}^{N}\left\|\partial_{x_{i}} u_{n}\right\|_{L^{p_{i}(\cdot)}}^{p_{m}^{-}} \\
& -\sum_{i \in \mathscr{F}_{1}}\left\|\partial_{x_{i}} u_{n}\right\|_{L^{p_{i}(\cdot)}}^{p_{m}^{-}} .
\end{aligned}
$$

Thus,

$$
\sum_{i=1}^{N} \int_{\Omega}\left|\partial_{x_{i}} u_{n}\right|^{p_{i}(x)} d x \geq \sum_{i=1}^{N}\left\|\partial_{x_{i}} u_{n}\right\|_{L^{p_{i}(\cdot)}}^{p_{m}^{-}}-N
$$

Definition 5. One defines the weak solution for problem (1) as a function $u \in X$ satisfying

$$
\begin{gathered}
\int_{\Omega} \sum_{i=1}^{N} a_{i}\left(x, \partial_{x_{i}} u\right) \partial_{x_{i}} v d x+\int_{\Omega}|u|^{p_{M}(x)-2} u v d x \\
-\int_{\Omega} f(x, u) v d x=0,
\end{gathered}
$$

for all $v \in X$.

We suppose the following hypotheses.

(F1) There exist $C>0$ and $q \in C_{+}(\bar{\Omega})$ with $p_{M}^{+}<q^{-} \leq$ $q^{+}<\bar{p}^{*}(x)$ for all $x \in \bar{\Omega}$, such that $f$ verifies

$$
|f(x, s)| \leq C\left(1+|s|^{q(x)-1}\right)
$$

for all $x \in \Omega$ and all $s \in \mathbb{R}$.

(F2) $\lim _{|t| \rightarrow \infty}\left(f(x, t) t /|t|^{p_{M}^{+}}\right)=\infty$ uniformly for $x \in \Omega$.

(F3) $f(x, s)=O\left(|s|^{p_{M}^{+}-1}\right)$ uniformly for $x \in \Omega$.

(F4) There exist two positive constants $\alpha$ and $\beta$ such that

$$
\psi_{1}(x, t) \leq \alpha \psi_{1}(x, s) \leq \beta \psi_{2}(x, s), \quad \forall 0 \leq t \leq s,
$$

where

$$
\begin{aligned}
& \psi_{1}(x, t)=f(x, t) t-p_{m}^{-} F(x, t), \\
& \psi_{2}(x, t)=f(x, t) t-p_{M}^{+} F(x, t),
\end{aligned}
$$

with $F(x, t)=\int_{0}^{t} f(x, s) d s$.

(A4) $p_{M}^{+} A_{i}(x, \xi) \geq a_{i}(x, \xi) \xi \geq p_{m}^{-} A_{i}(x, \xi) \geq 0$ for all $x \in \Omega$ and all $\xi \in \mathbb{R}$.

The function $f(x, t)=|t|^{q(x)-2} t$, where $p_{M}^{+}<q^{-} \leq q^{+}<$ $\bar{p} *(x)$ is an example of functions verifying the assumptions (F1)-(F4). In fact, we have

$$
\begin{gathered}
F(x, t)=\frac{|t|^{q(x)}}{q(x)}, \\
f(x, t) t=|t|^{q(x)},
\end{gathered}
$$

and then we get

$$
\begin{aligned}
& \psi_{1}(x, t)=\left(1-\frac{p_{m}^{-}}{q(x)}\right)|t|^{q(x)}, \\
& \psi_{2}(x, t)=\left(1-\frac{p_{M}^{+}}{q(x)}\right)|t|^{q(x)},
\end{aligned}
$$

which means that (F4) is satisfied since we have $\psi_{1}(x, t)$ which is nondecreasing in $t \geq 0$ and then $\psi_{1}(x, t) \leq \psi_{1}(x, s)$ when $0 \leq t \leq s$, so we take $\alpha=1$. Taking into account that $\psi_{1}, \psi_{2} \geq$ 0 , it follows that

$$
\frac{\psi_{1}(x, t)}{\psi_{2}(x, t)} \leq \frac{q^{+}-p_{m}^{-}}{q^{-}-p_{M}^{+}}=\beta .
$$

Obviously the other assumptions are held.

We report our main result. 
Theorem 6. Under conditions (A1)-(A4) and (F1)-(F4), problem (1) has at least a nontrivial weak solution.

The purpose of this work is to improve the results of the above-mentioned papers and many others, without assuming the Ambrosetti-Rabinowitz type conditions (A-R) used, for instance, in $[1-3,10]$, where in (A-R) there exist $\theta>p_{M}^{+}, A>0$ such that for any $x \in \Omega$ and $t \geq A$ we have

$$
0 \leq \theta F(x, t) \leq f(x, t) t
$$

In fact, it is known that (F4) is much weaker than the (A$\mathrm{R})$ condition in the constant exponent case (see, for instance, [22]). We will use the mountain pass theorem with Cerami condition which is weaker than (PS) condition used, for example, in $[4,6]$.

The energy functional corresponding to (1) is defined as $\phi: X \rightarrow \mathbb{R}$

$$
\begin{aligned}
\phi(u)= & \int_{\Omega} \sum_{i=1}^{N} A_{i}\left(x, \partial_{x_{i}} u\right) d x+\int_{\Omega} \frac{1}{p_{M}(x)}|u|^{p_{M}(x)} d x \\
& -\int_{\Omega} F(x, u) d x .
\end{aligned}
$$

By a standard argument, we can see that the functional $\phi$ is well defined and of class $C^{1}$, with its Gâteaux derivative being described by

$$
\begin{aligned}
\left\langle\phi^{\prime}(u), v\right\rangle= & \int_{\Omega} \sum_{i=1}^{N} a_{i}\left(x, \partial_{x_{i}} u\right) \partial_{x_{i}} v d x \\
& +\int_{\Omega}|u|^{p_{M}(x)-2} u v d x \\
& -\int_{\Omega} f(x, u) v d x
\end{aligned}
$$

for all $u, v \in X$.

Putting

$$
\begin{aligned}
I(u)= & \int_{\Omega} \sum_{i=1}^{N} A_{i}\left(x, \partial_{x_{i}} u\right) d x \\
& +\int_{\Omega} \frac{1}{p_{M}(x)}|u|^{p_{M}(x)} d x, \\
J(u)= & -\int_{\Omega} F(x, u) d x .
\end{aligned}
$$

Proposition 7. (i) By (A3), the functional $I^{\prime}$ is of $\left(S_{+}\right)$type; that is, if $u_{n} \rightarrow u$ and $\lim \sup _{n \rightarrow \infty}\left\langle I^{\prime}\left(u_{n}\right)-I^{\prime}(u), u_{n}-u\right\rangle$, then $u_{n} \rightarrow u$ in $X$.

(ii) From (F1), the functional $J^{\prime}$ is weakly strongly continuous; that is, $u_{n} \rightarrow u \Rightarrow J^{\prime}\left(u_{n}\right) \rightarrow J^{\prime}(u)$.

The proof of the first assertion (i) is similar to that in [2]. The second assertion is well known.

\section{Proof of the Main Result}

We will use the mountain pass theorem (see [23-25]), so we start by the condition of geometry in the form of the following lemma.

Lemma 8. (a) There exists $v \in X$ with $v>0$ such that $\phi(t v) \rightarrow$ $-\infty$ as $t \rightarrow \infty$.

(b) There exist $r, \sigma>0$ such that $\phi(u) \geq \sigma$ for $\|u\|=r$.

Proof. (a) From (F2), we may choose a constant $K>0$ such that

$$
F(x, s)>K|s|^{p_{M}^{+}} \quad \text { uniformly in } x \in \Omega,|s|>C_{K} .
$$

Let $t>1$ large enough and $v \in X$ with $v>0$, and from (A1) and (39) we get

$$
\begin{aligned}
& \phi(t v)=\int_{\Omega} \sum_{i=1}^{N} A_{i}\left(x, \partial_{x_{i}} t v\right) d x \\
& +\int_{\Omega} \frac{1}{p_{M}(x)}|t v|^{p_{M}(x)} d x-\int_{\Omega} F(x, t v) d x \\
& \leq t \sum_{i=1}^{N} \int_{\Omega} \overline{c_{i}}\left|b_{i}(x)\right|\left|\partial_{x_{i}} v\right| d x \\
& +t^{p_{M}^{+}} \sum_{i=1}^{N} \int_{\Omega} \overline{c_{i}} \frac{\left|\partial_{x_{i}} v\right|^{p_{i}(x)}}{p_{i}(x)} d x \\
& +t^{p_{M}^{+}} \int_{\Omega} \frac{1}{p_{M}(x)}|v|^{p_{M}(x)} d x \\
& -\int_{\Omega} F(x, t v) d x \\
& \leq \max _{1 \leq i \leq N} \overline{c_{i}} 2 t \sum_{i=1}^{N}\left\|b_{i}\right\|_{L^{p_{i}^{\prime}(\cdot)}(\Omega)}\left\|\partial_{x_{i}} v\right\|_{L^{p_{i}(\cdot)}(\Omega)} \\
& +t^{p_{M}^{+}} \max _{1 \leq i \leq N} \overline{c_{i}} \frac{1}{p_{m}^{-}} \sum_{i=1}^{N} \int_{\Omega}\left|\partial_{x_{i}} v\right|^{p_{i}(x)} d x \\
& +t^{p_{M}^{+}} \frac{1}{p_{M}^{-}} \int_{\Omega}|v|^{p_{M}(x)} d x \\
& -\int_{|t v|>C_{K}} F(x, t v) d x-\int_{|t v| \leq C_{K}} F(x, t v) d x \\
& \leq 2 t \max _{1 \leq i \leq N} \overline{c_{i}} \sum_{i=1}^{N}\left\|b_{i}\right\|_{L^{p_{i}^{\prime}(\cdot)}(\Omega)}\left\|\partial_{x_{i}} v\right\|_{L^{p_{i}(\cdot)}(\Omega)} \\
& +t^{p_{M}^{+}} \frac{\max _{1 \leq i \leq N} \overline{c_{i}}}{p_{m}^{-}} \sum_{i=1}^{N} \int_{\Omega}\left|\partial_{x_{i}} v\right|^{p_{i}(x)} d x \\
& +t^{p_{M}^{+}} \frac{1}{p_{M}^{-}} \int_{\Omega}|v|^{p_{M}(x)} d x-K t^{p_{M}^{+}} \int_{\Omega}|v|^{p_{M}^{+}} d x \\
& -\int_{|t v| \leq C_{K}} F(x, t v) d x
\end{aligned}
$$




$$
\begin{aligned}
& \leq 2 t \max _{1 \leq i \leq N} \overline{c_{i}} \sum_{i=1}^{N}\left\|b_{i}\right\|_{L^{p_{i}^{\prime}(\cdot)}(\Omega)}\left\|\partial_{x_{i}} v\right\|_{L^{p_{i}(\cdot)}(\Omega)} \\
& +t^{p_{M}^{+}} \frac{\max _{1 \leq i \leq N} \bar{c}_{i}}{p_{m}^{-}} \sum_{i=1}^{N} \int_{\Omega}\left|\partial_{x_{i}} v\right|^{p_{i}(x)} d x \\
& +t^{p_{M}^{+}} \frac{1}{p_{M}^{-}} \int_{\Omega}|v|^{p_{M}(x)} d x-K t^{p_{M}^{+}} \int_{\Omega}|v|^{p_{M}^{+}} d x \\
& +C_{1},
\end{aligned}
$$

where $C_{1}>0$ is a constant, taking $K$ sufficiently large to ensure that

$$
\begin{gathered}
\frac{\max _{1 \leq i \leq N} \bar{c}_{i}}{p_{m}^{-}} \sum_{i=1}^{N} \int_{\Omega}\left|\partial_{x_{i}} v\right|^{p_{i}(x)} d x+\frac{1}{p_{M}^{-}} \int_{\Omega}|v|^{p_{M}(x)} d x \\
\quad-K \int_{\Omega}|v|^{p_{M}^{+}} d x<0
\end{gathered}
$$

which implies that

$$
\phi(t v) \longrightarrow-\infty \quad \text { as } t \longrightarrow+\infty \text {. }
$$

(b) By (A2), for $\|u\|<1$, we have

$$
\begin{aligned}
\phi(u) \geq & \frac{\min _{1 \leq i \leq N} d_{i}}{p_{M}^{+}} \int_{\Omega} \sum_{i=1}^{N}\left|\partial_{x_{i}} u\right|^{p_{i}(x)} d x \\
& +\frac{1}{p_{M}^{+}} \int_{\Omega}|u|^{p_{M}(x)} d x-\int_{\Omega} F(x, u) d x \\
\geq & \frac{\min _{1 \leq i \leq N} d_{i}}{p_{M}^{+}} \sum_{i=1}^{N}\left\|\partial_{x_{i}} u\right\|_{L^{p_{i}(\cdot)}(\Omega)}^{p^{+}}+\frac{1}{p_{M}^{+}}\|u\|_{L^{p_{M}^{(\cdot)}(\Omega)}}^{p^{+}} \\
& -\int_{\Omega} F(x, u) d x \\
\geq & \frac{\min _{1 \leq i \leq N} d_{i}}{(N+1)^{p_{m}^{+}} p_{M}^{+}}\|u\|^{p_{M}^{+}}-\int_{\Omega} F(x, u) d x .
\end{aligned}
$$

On the other side, from (F1),

$$
\begin{aligned}
&|f(x, u)| \leq \varepsilon|u|^{p_{M}^{+}-1}+C(\varepsilon)|u|^{q(x)-1} \\
& \forall(x, u) \in \Omega \times \mathbb{R} .
\end{aligned}
$$

By the continuous embedding from $X$ into $L^{q(x)}(\Omega)$ and $L^{p_{M}^{+}}(\Omega)$, there exist $c_{1}, c_{2}>0$, such that

$$
\begin{gathered}
\|u\|_{L^{p_{M}^{+}(\Omega)}} \leq c_{1}\|u\|, \\
\|u\|_{L^{q^{+}(\Omega)}} \leq c_{2}\|u\|, \\
\|u\|_{L^{q^{-}}(\Omega)} \leq c_{2}\|u\|
\end{gathered}
$$

for all $u \in X$. Hence,

$$
\begin{aligned}
\int_{\Omega} F(x, u) d x \leq & \int_{\Omega} \frac{\varepsilon}{p_{M}^{+}}|u|^{p_{M}^{+}} d x \\
& +\int_{\Omega} \frac{C(\varepsilon)}{q(x)}|u|^{q(x)} d x \\
\leq & \varepsilon c_{1}^{p_{M}^{+}}\|u\|^{p_{M}^{+}}+c_{2}^{q^{-}} \frac{C(\varepsilon)}{q^{-}}\|u\|^{q^{-}}
\end{aligned}
$$

for all $x \in \Omega$ and all $u \in \mathbb{R}$.

Therefore,

$$
\begin{aligned}
& \phi(u) \geq\left(\frac{\min _{1 \leq i \leq N}\left(d_{i}, 1\right)}{2(N+1)^{p_{m}^{+}} p_{M}^{+}}-C(\varepsilon) c_{2}^{q^{-}}\|u\|^{q^{-}-p_{M}^{+}}\right. \\
& \left.-\varepsilon c_{1}^{p_{M}^{+}}\right)\|u\|^{p_{M}^{+}}
\end{aligned}
$$

since $1<p_{M}^{+}<q^{-}$. Then for $r$ sufficiently small, we take $\sigma>0$ such that

$$
\phi(u) \geq \sigma, \quad \forall u \in X \text { with }\|u\|=r
$$

Definition 9. A sequence $\left(z_{n}\right)$ is called a Cerami sequence if $\phi\left(z_{n}\right)$ is bounded and $\left(1+\left\|z_{n}\right\|\right) \phi^{\prime}\left(z_{n}\right) \rightarrow 0$.

Lemma 10. If $c \in \mathbb{R}$, then any sequence of Cerami $(C)_{c}$ of $\phi$ is bounded.

Proof. Let $\left(u_{n}\right)_{n}$ be a $(C)_{c}$ sequence of $\phi$. We claim that $\left(u_{n}\right)_{n}$ is bounded; otherwise, up to a subsequence, we may assume that

$$
\begin{aligned}
\phi\left(u_{n}\right) & \longrightarrow c, \\
\left\|u_{n}\right\| & \longrightarrow+\infty, \\
\phi^{\prime}\left(u_{n}\right) & \longrightarrow 0 .
\end{aligned}
$$

Putting $\omega_{n}=u_{n} /\left\|u_{n}\right\|$, up to a subsequence, we have $\omega_{n} \rightarrow \omega$ in $X, \omega_{n} \rightarrow \omega$ in $L^{q(x)}(\Omega)$ and in $L^{p_{M}^{+}}(\Omega), \omega_{n}(x) \rightarrow \omega(x)$. Almost everywhere $x \in \Omega$.

Here, two cases appear, when $\omega \neq \equiv 0$, since we know that $\left\langle\phi^{\prime}\left(u_{n}\right), u_{n}\right\rangle=o\left(\left\|u_{n}\right\|\right)$, that means

$$
\begin{gathered}
\int_{\Omega} \sum_{i=1}^{N} a_{i}\left(x, \partial_{x_{i}} u_{n}\right) \partial_{x_{i}} u_{n} d x+\int_{\Omega}\left|u_{n}\right|^{p_{M}(x)} d x \\
-\int_{\Omega} f\left(x, u_{n}\right) u_{n} d x=0 .
\end{gathered}
$$

Dividing (50) by $\left\|u_{n}\right\|^{p_{M}^{+}}$, by using (A1), a straightforward computation leads to

$$
\int_{\Omega} \frac{f\left(x, u_{n}\right) u_{n}}{\left\|u_{n}\right\|^{p_{M}^{+}}} d x<\infty .
$$


Meanwhile, in view of condition (F2) and Fatou's lemma,

$$
\begin{aligned}
\int_{\Omega} \frac{f\left(x, u_{n}\right) u_{n}}{\left\|u_{n}\right\|^{p_{M}^{+}}} d x & =\int_{\Omega} \frac{f\left(x, u_{n}\right) u_{n}\left|\omega_{n}\right|^{p_{M}^{+}}}{\left|u_{n}\right|^{p_{M}^{+}}} d x \\
& \longrightarrow \infty
\end{aligned}
$$

which is contradictory.

In the case when $\omega \equiv 0$, we choose a sequence $t_{n} \in[0,1]$ satisfying

$$
\phi\left(t_{n} u_{n}\right)=\max _{t_{n} \in[0,1]} \phi\left(t u_{n}\right)
$$

If $\omega \equiv 0$, since $\omega_{n} \rightarrow 0$ in $L^{q(x)}(\Omega)$ and $|F(x, t)| \leq C(1+$ $\left.|t|^{q(x)}\right)$, by the continuity of the Nemitskii operator, we see that $F\left(\cdot, \omega_{n}\right) \rightarrow 0$ in $L^{1}(\Omega)$ as $n \rightarrow+\infty$; therefore,

$$
\lim _{n \rightarrow \infty} \int_{\Omega} F\left(x, \omega_{n}\right) d x=0
$$

Given $m>0$, since, for $n$ large enough, we have $\left\|u_{n}\right\|^{-1}\left(2 m N p_{M}^{+}\right)^{1 / p_{m}^{-}} \in(0,1)$, using (54) with $R=$ $\left(2 m N p_{M}^{+}\right)^{1 / p_{m}^{-}}$, from assumption (A2), and, considering Remark 4, we get

$$
\begin{aligned}
& \phi\left(t_{n} u_{n}\right) \geq \phi\left(\frac{R}{\left\|u_{n}\right\|} u_{n}\right)=\phi\left(R \omega_{n}\right) \\
& =\int_{\Omega} \sum_{i=1}^{N} A_{i}\left(x, \partial_{x_{i}} R \omega_{n}\right) d x \\
& +\int_{\Omega} \frac{1}{p_{M}(x)}\left|R \omega_{n}\right|^{p_{M}(x)} d x-\int_{\Omega} F\left(x, R \omega_{n}\right) d x \\
& \geq \frac{\min _{1 \leq i \leq N} d_{i}}{p_{M}^{+}}\left(\sum_{i=1}^{N}\left\|\partial_{x_{i}} R \omega_{n}\right\|_{L^{p_{i}(\cdot)}(\Omega)}^{p_{-}^{-}}\right. \\
& \left.+\left\|R \omega_{n}\right\|_{L^{p^{(\cdot)}(\Omega)}}^{p^{-}}-N\right)-\int_{\Omega} F\left(x, R \omega_{n}\right) d x \geq \frac{1}{p_{M}^{+}} \\
& \cdot \min \left\{1, \min _{1 \leq i \leq N} d_{i}\right\}\left(\frac{\left\|R \omega_{n}\right\|^{p_{m}^{-}}}{p_{M}^{+}}-N\right) \\
& -\int_{\Omega} F\left(x, R w_{n}\right) d x \geq C_{0}\left(\frac{R^{p_{m}^{-}}}{p_{M}^{+}}-N\right) \\
& -\int_{\Omega} F\left(x, R w_{n}\right) d x>m .
\end{aligned}
$$

Thereby, $\phi\left(t_{n} u_{n}\right) \rightarrow+\infty$. On the other hand, we know that $\phi(0)=0, \phi\left(u_{n}\right) \rightarrow c$, so we can deduce that

$$
\begin{gathered}
\left.t_{n} \in\right] 0,1[ \\
\left\langle\phi^{\prime}\left(t_{n} u_{n}\right), t_{n} u_{n}\right\rangle=\left.t_{n} \frac{d}{d t}\right|_{t=t_{n}} \phi\left(t u_{n}\right)=0 .
\end{gathered}
$$

It yields

$$
\phi\left(t_{n} u_{n}\right)-\frac{1}{p_{m}^{-}} \phi^{\prime}\left(t_{n} u_{n}\right)\left(t_{n} u_{n}\right) \longrightarrow+\infty
$$

Therefore,

$$
\begin{aligned}
& \int_{\Omega} \sum_{i=1}^{N} A_{i}\left(x, \partial_{x_{i}} t_{n} u_{n}\right) d x \\
& \quad-\frac{1}{p_{m}^{-}} \int_{\Omega} \sum_{i=1}^{N} a_{i}\left(x, \partial_{x_{i}} t_{n} u_{n}\right) \partial_{x_{i}} t_{n} u_{n} d x \\
& \quad+\int_{\Omega} \frac{1}{p_{M}(x)}\left|t_{n} u_{n}\right|^{p_{M}(x)} d x \\
& \quad-\frac{1}{p_{m}^{-}} \int_{\Omega}\left|t_{n} u_{n}\right|^{p_{M}(x)} d x-\int_{\Omega} F\left(x, t_{n} u_{n}\right) d x \\
& \quad+\int_{\Omega}\left(\frac{1}{p_{m}^{-}} f\left(x, t_{n} u_{n}\right) t_{n} u_{n}\right) d x \longrightarrow \infty,
\end{aligned}
$$

so we get

$$
\int_{\Omega}\left(\frac{1}{p_{m}^{-}} f\left(x, t_{n} u_{n}\right)\left(t_{n} u_{n}\right)-F\left(x, t_{n} u_{n}\right)\right) d x
$$

$\longrightarrow+\infty$.

Moreover,

$$
\begin{aligned}
\phi\left(u_{n}\right) & =\phi\left(u_{n}\right)-\frac{1}{p_{M}^{+}} \phi^{\prime}\left(u_{n}\right)\left(u_{n}\right) \\
& \geq \int_{\Omega}\left(\frac{1}{p_{M}^{+}} f\left(x, u_{n}\right) u_{n}-F\left(x, u_{n}\right)\right) d x
\end{aligned}
$$

From (A2) and (F4), there exist $\alpha, \beta>0$ such that

$$
\begin{aligned}
& \phi\left(u_{n}\right) \geq \int_{\Omega}\left(\frac{1}{p_{M}^{+}} f\left(x, u_{n}\right) u_{n}-F\left(x, u_{n}\right)\right) d x \\
& \geq \alpha \int_{\Omega}\left(\frac{1}{p_{m}^{-}} f\left(x, u_{n}\right)\left(u_{n}\right)-F\left(x, u_{n}\right)\right) d x \\
& \quad \geq \alpha \beta \int_{\Omega}\left(\frac{1}{p_{m}^{-}} f\left(x, t_{n} u_{n}\right)\left(t_{n} u_{n}\right)-F\left(x, t_{n} u_{n}\right)\right) d x .
\end{aligned}
$$

Hence, $\phi\left(u_{n}\right) \rightarrow+\infty$, which is impossible.

Proof of Theorem 6. According to Lemma 8 and Lemma 10, we are to apply the mountain pass theorem, so seeing that the sequence $\left(u_{n}\right)_{n}$ (in Lemma 10 ) is strongly convergent to $u \in X$ remains and it will be done.

Now, because the Banach space $X$ is reflexive (cf. $[2,3]$ ), and regarding the boundedness of $\left(u_{n}\right)_{n}$ in $X$, there exists $u \in X$ such that $u_{n} \rightarrow u$. Since $\phi^{\prime}$ is the sum of $\left(S_{+}\right)$ type maps $I^{\prime}$ and $J^{\prime}$ which is weakly strongly continuous (cf. Proposition 7), $\phi^{\prime}$ is also of $\left(S_{+}\right)$type. Thus, $u_{n} \rightarrow u$ in $X$. 


\section{Conflict of Interests}

The author declares that there is no conflict of interests regarding the publication of this paper.

\section{Acknowledgment}

The author would like to thank the referee for the suggestions and helpful comments which improved the presentation of the original paper.

\section{References}

[1] C. O. Alves and A. El Hamidi, "Existence of solution for an anisotropic equation with critical exponent," Nonlinear Analysis: Theory, Methods \& Applications, vol. 4, pp. 611-624, 2005.

[2] M.-M. Boureanu, P. Pucci, and V. D. Radulescu, "Multiplicity of solutions for a class of anisotropic elliptic equations with variable exponent," Complex Variables and Elliptic Equations, vol. 56, no. 7-9, pp. 755-767, 2011.

[3] M.-M. Boureanu, "Critical point methods in degenerate anisotropic problems with variable exponent," Studia Universitatis Babes-Bolyai, Mathematica, vol. 55, no. 4, pp. 27-39, 2010.

[4] N. T. Chung and H. Q. Toan, "On a class of anisotropic elliptic equations without Ambrosetti-Rabinowitz type conditions," Nonlinear Analysis. Real World Applications, vol. 16, pp. 132-145, 2014.

[5] F. C. Cîrstea and J. Vétois, "Fundamental solutions for anisotropic elliptic equations: existence and a priori estimates," Communications in Partial Differential Equations, vol. 40, no. 4, pp. 727-765, 2015.

[6] D. S. Dumitru, "Multiplicity of solutions for a nonlinear degenerate problem in anisotropic variable exponent spaces," Bulletin of the Malaysian Mathematical Sciences Society, vol. 36, no. 1, pp. 117-130, 2013.

[7] A. El Hamidi and J. Vétois, "Sharp Sobolev asymptotics for critical anisotropic equations," Archive for Rational Mechanics and Analysis, vol. 192, no. 1, pp. 1-36, 2009.

[8] X. Fan, "Anisotropic variable exponent Sobolev spaces and $\overrightarrow{p(x)}$-Laplacian equations," Complex Variables and Elliptic Equations, vol. 56, no. 7-9, pp. 623-642, 2011.

[9] I. Fragalà, F. Gazzola, and B. Kawohl, "Existence and nonexistence results for anisotropic quasilinear elliptic equations," Annales de l'Institut Henri Poincaré C: Non Linear Analysis, vol. 21, no. 5, pp. 715-734, 2004.

[10] B. Kone, S. Ouaro, and S. Traore, "Weak solutions for anisotropic nonlinear elliptic equations with variable exponents," Electronic Journal of Differential Equations, vol. 2009, no. 144, pp. 1-11, 2009.

[11] A. Ourraoui, "On a nonlocal $\vec{p}(x)$-Laplacian equations via genus theory," Rivista di Matematica della Università di Parma, In press.

[12] J. Vétois, "Strong maximum principles for anisotropic elliptic and parabolic equations," Advanced Nonlinear Studies, vol. 12, no. 1, pp. 101-114, 2012.

[13] J. Vétoiss, "Existence and regularity for critical Anisotropic equations with critical directions," Advances in Differential Equations, vol. 16, no. 1-2, pp. 61-83, 2011.

[14] J. Batt and Y. Li, "The positive solutions of the Matukuma equation and the problem of finite radius and finite mass,"
Archive for Rational Mechanics and Analysis, vol. 198, no. 2, pp. 613-675, 2010.

[15] A. J. Simmonds, "Electro-rheological valves in a hydraulic circuit," IEE Proceedings D: Control Theory and Applications, vol. 138, no. 4, pp. 400-404, 1991.

[16] S. N. Antontsev and J. F. Rodrigues, "On stationary thermorheological viscous flows," Annali dell'Università di Ferrara Sezione VII. Scienze Matematiche, vol. 52, pp. 19-36, 2006.

[17] V. V. Zhikov, "Averaging of functionals in the calculus of variations and elasticity," Mathematics of the USSR-Izvestiya, vol. 29, no. 1, pp. 33-66, 1987.

[18] Y. Chen, S. Levine, and M. Rao, "Variable exponent, linear growth functionals in image restoration," SIAM Journal on Applied Mathematics, vol. 66, no. 4, pp. 1383-1406, 2006.

[19] M. Bendahmane, M. Langlais, and M. Saad, "On some anisotropic reaction-diffusion systems with $L^{1}$-data modeling the propagation of an epidemic disease," Nonlinear Analysis: Theory, Methods \& Applications, vol. 54, no. 4, pp. 617-636, 2003.

[20] D. E. Edmunds and J. R'akosnk, "Sobolev embedding with variable exponent," Studia Mathematica, vol. 143, no. 3, pp. 267293, 2000.

[21] O. Kováčik and J. Rákosník, "On spaces $L^{p(x)}$ and $W^{k, p(x), "}$ Czechoslovak Mathematical Journal, vol. 41, pp. 592-618, 1991.

[22] D. Geng, "Infinitely many solutions of $p$-Laplacian equations with limit subcritical growth," Applied Mathematics and Mechanics, vol. 28, no. 10, pp. 1373-1382, 2007.

[23] P. Pucci, "Geometric description of the mountain pass critical points," in Contemporary Mathematicians, vol. 2, pp. 469-471, Birkhuser, Basel, Switzerland, 2014.

[24] P. Pucci and V. Radulescu, "The impact of the mountain pass theory in nonlinear analysis: a mathematical survey," Bollettino dell'Unione Matematica Italiana Series IX, vol. 3, pp. 543-584, 2010 .

[25] M. Willem, Minimax Theorems, Birkhuser, 1996. 


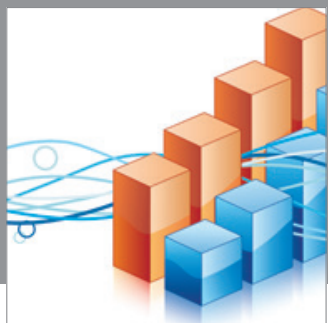

Advances in

Operations Research

mansans

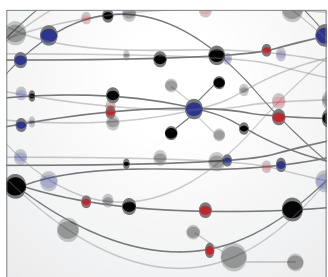

The Scientific World Journal
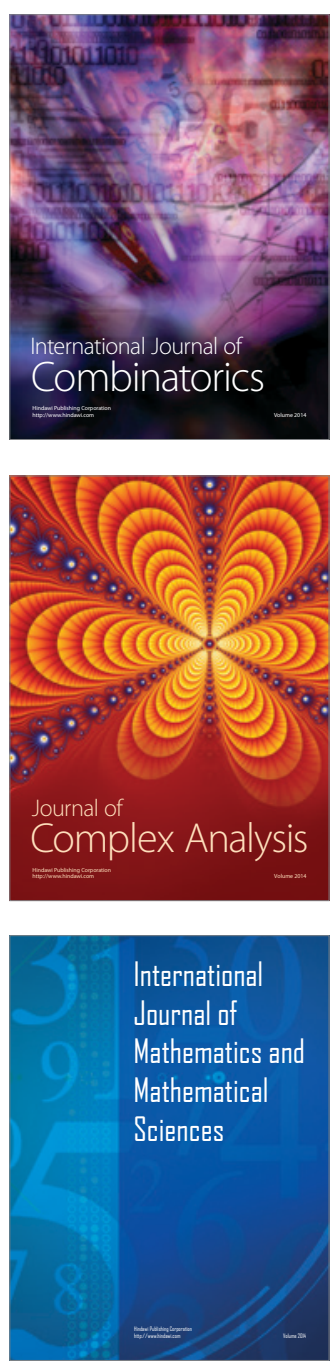
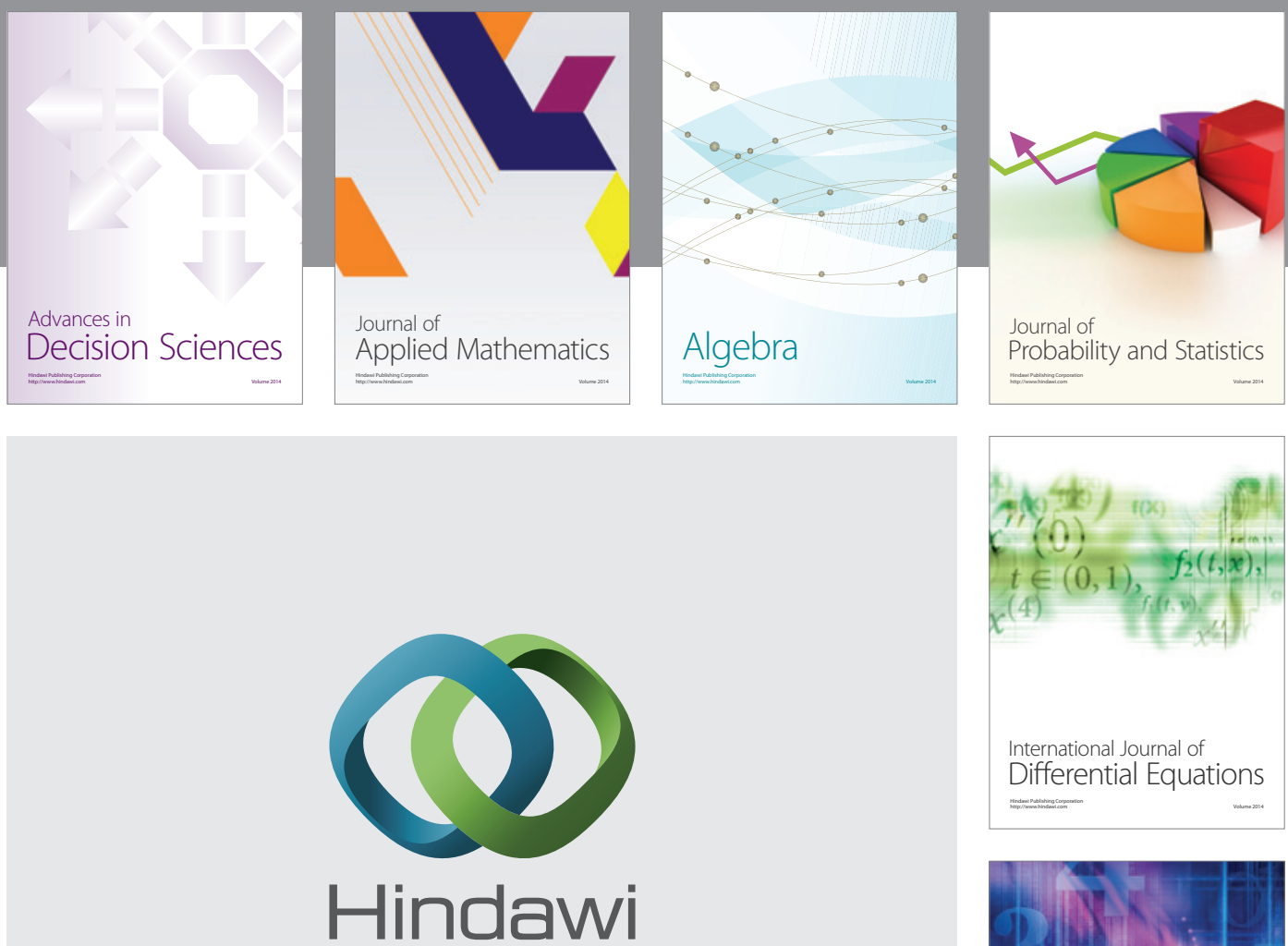

Submit your manuscripts at http://www.hindawi.com
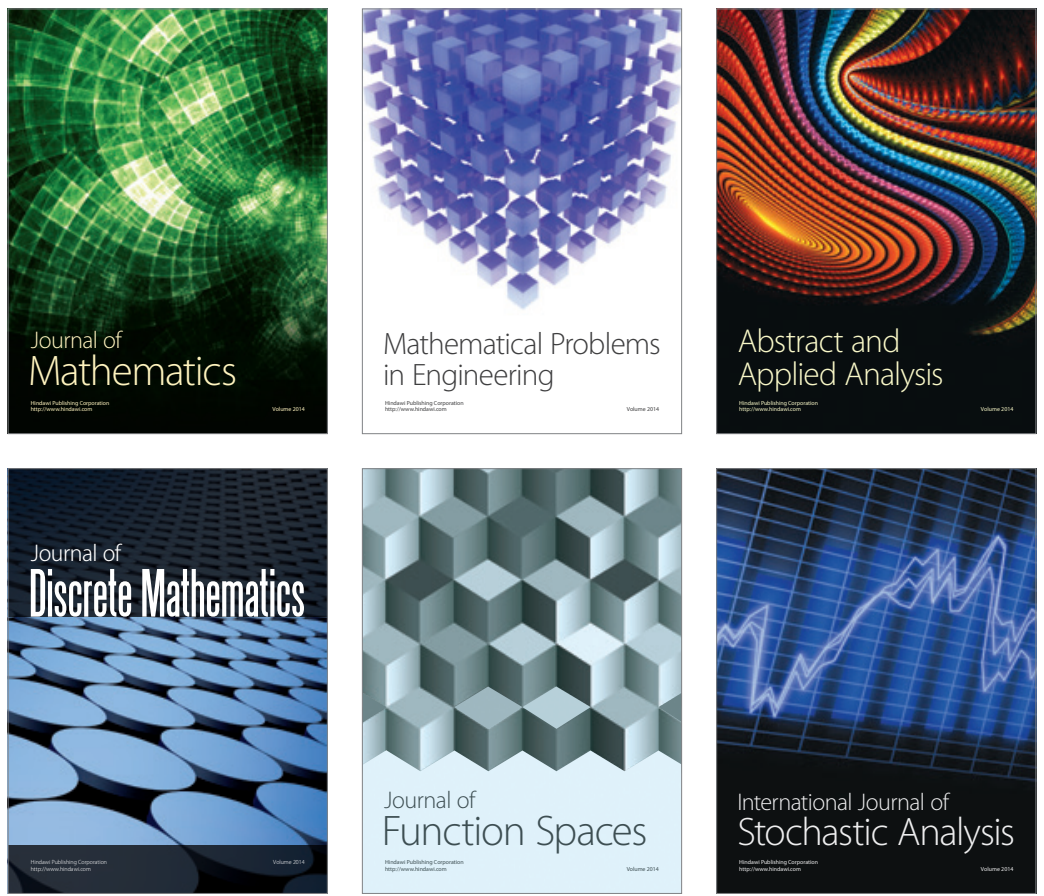

Journal of

Function Spaces

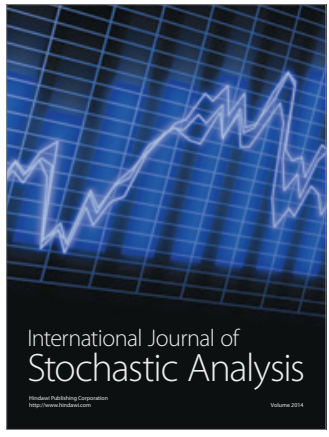

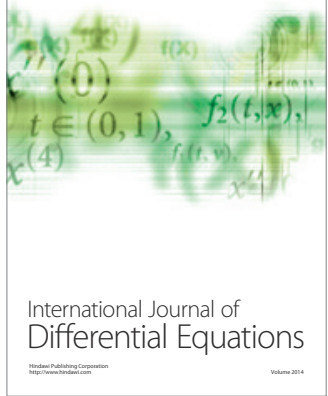
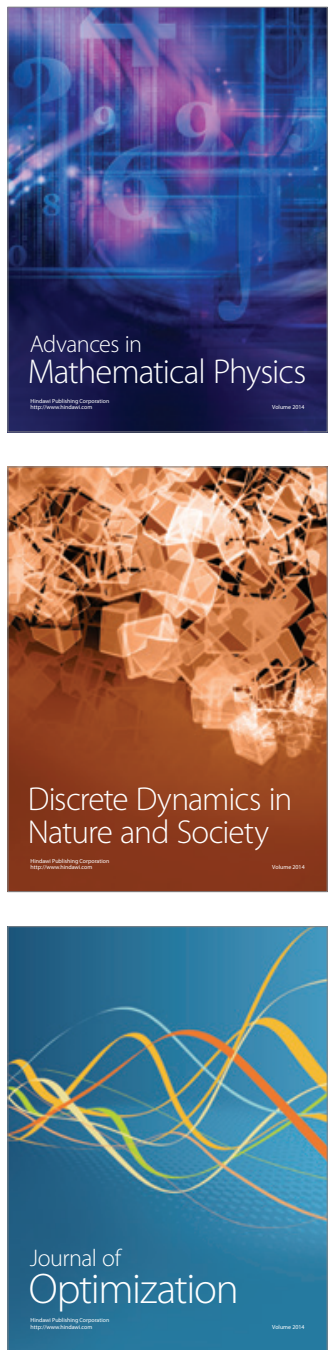